\title{
REQUIREMENTS ON NEEDED FREQUENCY BANDWIDTH DEPENDING ON PULSE WAVEFORMS AND THEIR ALLOWED DISTORTION
}

\author{
Milan Sigmund — Lubomir Brancik *
}

\begin{abstract}
This paper deals with pulse signals influenced by loss of energy in high frequency band. Five types of pulses were tested and evaluated under various conditions. Achieved results can be helpful for some specific tasks in signal transmission. An example presents highest frequency of periodic pulse signals processed on printed circuit board.

K e y w ords: pulse signal, limited channel bandwidth, energy and power
\end{abstract}

\section{INTRODUCTION}

Energy is one of the basic features characterizing realworld signals. Physically, the energy of a signal represents its capacity to perform work. In different fields of scientific research (e.g., pattern recognition, feature extraction, biomedical signal processing, etc.), signal energy (mostly short-time energy) needs to be detected, analyzed or eliminated [1]. Many practical applications of signal energy can be found in the field of pulse signal processing. For instance, the study [2] describes a method to determine the attenuation of the signal energy through the region of the body in the heart area using Gaussian pulses whose pulse width varies from 1 to $10 \mathrm{GHz}$. In the photoplethysmography, a correlation of changes in the pulse width of the measured photoplethysmographic signal with changes in peripheral vascular resistance was found in [3]. The paper [4] provides an overview of methods dealing with energy concentration in the time-frequency domain. The results of the literature review indicate that using energy concentration as a feature is a very powerful tool and has been utilized in numerous applications. Besides the low voltage applications mentioned above, pulse signals are analyzed and utilized also in high voltage applications. For instance, the paper [5] deals with the use of pulse signals in large capacity cascaded battery energy storage system. The signal energy plays very important role also in communication systems, such as wireless networks [6], radars [7] as well as internet of things [8], whether are investigated the radio signals themselves (source signals, transmitted signals, received signals) or are observed the surrounding signals (noise, background) for optimization of the signal processing.

In order to right evaluate all features derived from signal energy, the potential energy must not be affected by any operation during signal processing. Our study deals with five types of pulse waveform and their robustness to the effect of spectral limitation caused, for example, by transmission channel.

\section{CALCULATING SIGNAL POWER}

In our study, signal is represented by voltage which is the most common physical type of signals applied in electronics and communications. The pulse energy or power was calculated using the Parsevals theorem [9] which relates values obtained in time and frequency domains. The mean power $P$ in periodic signals with repetition period $T$ is given by either of the expressions

$$
P=\frac{1}{T} \int_{0}^{T} s^{2}(t) d t=\sum_{k=-\infty}^{\infty}\left|c_{k}\right|^{2},
$$

where $s(t)$ is a pulse wave and the absolute value $\left|c_{k}\right|$ gives magnitude of the complex Fourier coefficients (magnitudes of all coefficients represent amplitude spectrum). Single coefficients $c_{k}$ correspond to the harmonic frequencies of the fundamental frequency $\omega_{1}$ as

$$
c_{k}=k \omega_{1}=\omega_{k}
$$

where $\omega_{1}=2 \pi / T$. When $k=0$, the coefficient $c_{0}$ stands for the DC component, ie zero-frequency component contained in signal $s(t)$. More details on Fourier coefficients including their computing and properties can be found, for example, in [10].

Precisely, (1) defines mean signal power $P$ in watts only when the signal $s(t)$ in volts is applied across a resistance of $R=1 \Omega$. Since the resistance value is unity the dissipated power may be referred to as normalized power. For an isolated pulse (having finite energy) the normalized energy is similarly defined. In the following text we shall refer to it simply as power or energy, with

* Brno University of Technology, Department of Radio Electronics, Technicka 12, 61600 Brno, sigmund@feec.vutbr.cz 

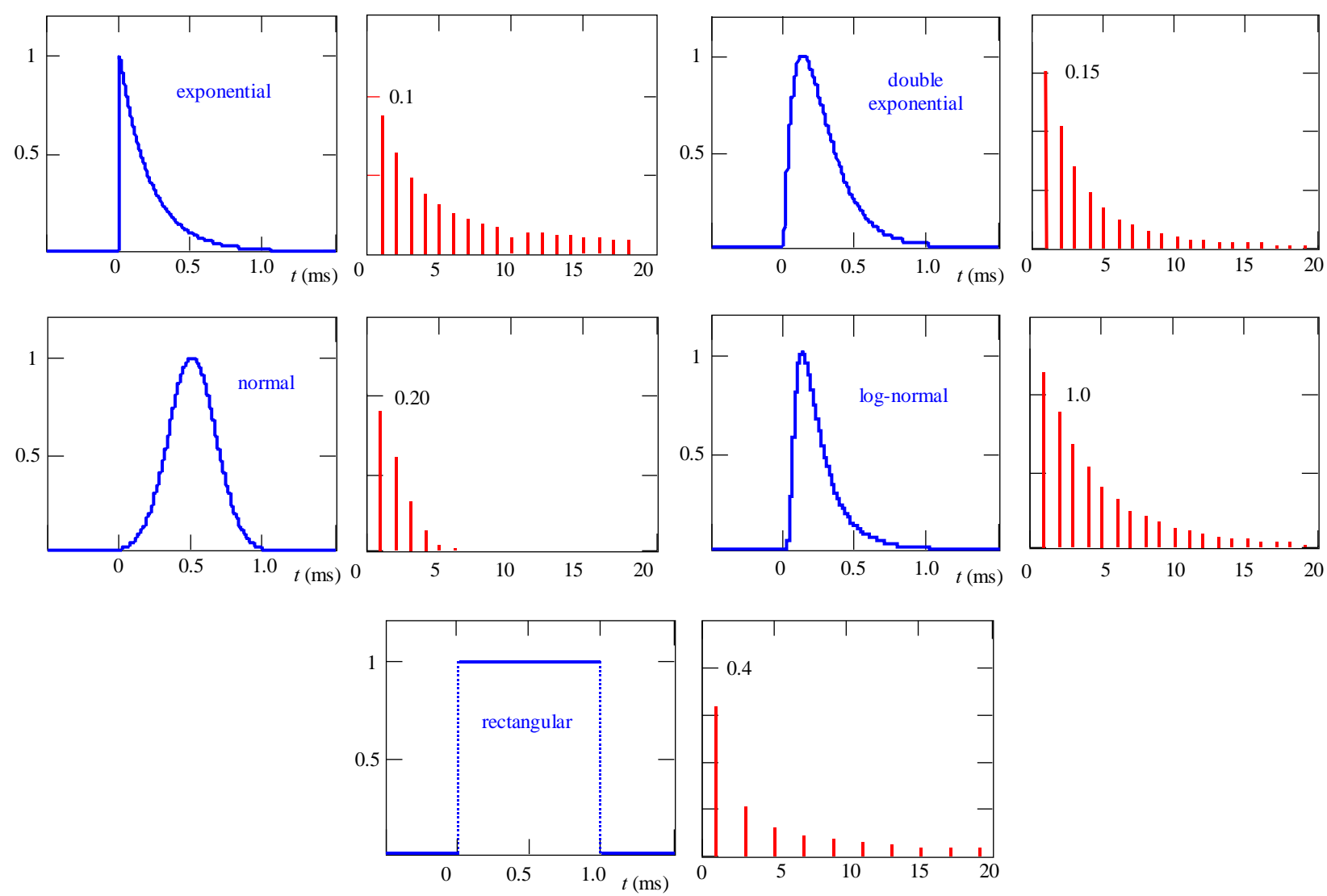

Fig. 1. Waveforms (left) and corresponding spectra (right) without DC component of analyzed pulses

the implicit assumption that it is the normalized power or normalized energy.

\section{PULSES IN COMPARISON}

An endless variety of different pulse signals is possible. We have analyzed chosen pulses with waveforms based on several commonly used functions such as exponential, logarithm, and quadrature. These pulses are easy to generate using both discrete and analogue circuits. In the analysis were taken into account five signals as follows: $s_{1}(t)$ - single exponential pulse; $s_{2}(t)$ - double exponential pulse; $s_{3}(t)$ - normal (Gaussian) pulse; $s_{4}(t)$ - logarithmic-normal pulse; and additionally rectangular pulse $r(t)$ for comparison purposes.

$$
\begin{gathered}
s_{1}(t)=A_{1} \exp \left(-\frac{t}{\tau_{1}}\right) \\
s_{2}(t)=A_{2}\left[\exp \left(-\frac{\alpha t}{\tau_{2}}\right)-\exp \left(-\frac{\beta t}{\tau_{2}}\right)\right] \\
s_{3}(t)=A_{3} \exp \left(-\left(\frac{t-\mu}{\tau_{3}}\right)^{2}\right) \\
s_{4}(t)=A_{4} \exp \left(-\left(\frac{\ln (t)-\mu}{\tau_{4}}\right)^{2}\right)
\end{gathered}
$$

$$
r(t)= \begin{cases}A & \text { for } t \in\langle 0 ; \eta T\rangle, \quad \eta \in(0 ; 1) \\ 0 & \text { otherwise }\end{cases}
$$

The pulse waveforms are derived from some probability distributions. An adequate background may be found, for example, in [11]. In (3) to (6), $A$ is the scale factor, $\tau$ is the time delay, $\mu$ represents time location, and $\alpha, \beta$ are specific constants. In (7), $\eta$ represents a duty cycle considering a completed period $T$ of pulse train. The values of all mentioned parameters were adjusted such as creating pulses of unit peak and duration of one millisecond. In case of asymptotic falling edges, a fall below $1 \%$ is considered to be the endpoint for determining the pulse width. Figure 1 shows the waveforms of single pulses $s_{1}(t)$ to $s_{4}(t)$ as well as $r(t)$ and their amplitude spectra for the first twenty coefficients $c_{k}$. The spectra were obtained by short-time Fourier transform [12].

\section{RESULTS}

Our experiments were focused on loss of energy together with features derived from the loss such as drop in pulse height and change of pulse shape. The cause of energy loss in pulses is limited spectral width of channel which transmits the pulses. Signal models were tested using the mathematical software Mathcad [13]. Three criteria were applied in investigating the effect of filtered out high spectral components from signal. First, pulse height 


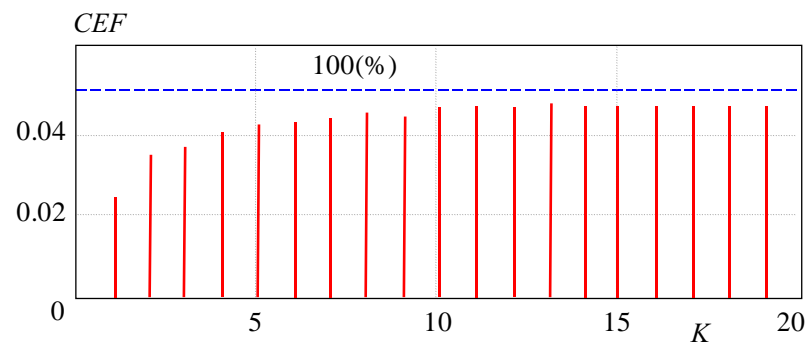

Fig. 2. Cumulative energy function for exponential pulse with incremented number of harmonics

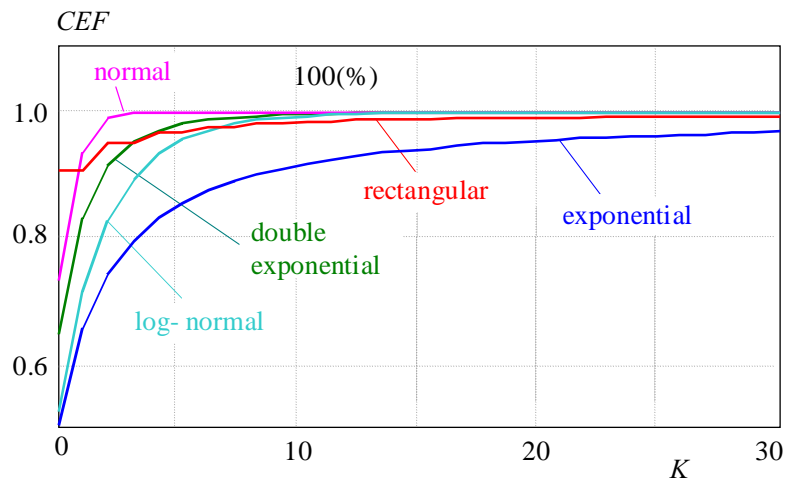

Fig. 3. Normalized cumulative energy functions of all types of pulses

was measured as the highest peak in the waveform. Second, the waveform-matching parameter (in percent) was estimated using the ratio of areas

$$
\rho=\frac{\int_{0}^{T}|s(t)-x(t)| d t}{\int_{0}^{T}|s(t)| d t} 100,
$$

where $s(t)$ is the original pulse (here substituting all types of pulses) and $x(t)$ stands for appropriate deformed pulse after filtering. Last, loss of energy was calculated by means of cumulative energy function

$$
C E F(K)=\left|c_{0}\right|^{2}+2\left|c_{1}\right|^{2}+\ldots+2\left|c_{k}\right|^{2}+\ldots+2\left|c_{K}\right|^{2}
$$

where $K$ is the continuously incremented total number of Fourier coefficients, ie harmonic components, considered in the summation. Because the complex conjugate coefficients $c_{k}$ and $c_{-k}$ have equal magnitude, the terms $\left|c_{k}\right|^{2}$ for $k \geq 1$ are doubled in (9). The curve of $C E F(K)$ for exponential pulse can be seen in Fig. 2. Normalized curves $C E F(K)$ for all pulses are compared in Fig. 3. The total pulse energy marked as the line $100 \%$ was calculated from waveform of the original pulses according the left side of (1).

For three thresholds of changes (namely 60, 90, and 98 percent) in the defined parameters, number of harmonics (including DC component) needed to be present in a wave of each pulse signal was investigated. Achieved results are summarized in Tabs. 1, 2, and 3.
Table 1. Changes of peak level

\begin{tabular}{llcc}
\hline Pulse signal & \multicolumn{2}{c}{$\begin{array}{c}\text { Number of harmonics } \\
\text { needed }\end{array}$} \\
\hline \multicolumn{1}{c}{$\%$} & 60 & 90 & peak \\
\hline exponential & 6 & 21 & 39 \\
double exponential & 4 & 8 & 11 \\
normal & 3 & 4 & 5 \\
log-normal & 3 & 6 & 8 \\
rectangular & 2 & 2 & 2 \\
\hline
\end{tabular}

Table 2. Changes of signal shape

\begin{tabular}{lccc}
\hline Pulse signal & \multicolumn{3}{c}{$\begin{array}{c}\text { Number of harmonics } \\
\text { needed for }\end{array}$} \\
\hline$\%$ & 60 & 90 & shape \\
\hline exponential & 8 & 41 & 266 \\
double exponential & 6 & 17 & 44 \\
normal & 3 & 5 & 6 \\
log-normal & 5 & 8 & 10 \\
rectangular & 2 & 12 & 84 \\
\hline
\end{tabular}

\begin{tabular}{|c|c|c|c|}
\hline \multirow{2}{*}{$\begin{array}{c}\text { Pulse signal } \\
\% \\
\end{array}$} & \multicolumn{3}{|c|}{$\begin{array}{l}\text { Number of harmonics } \\
\text { covering the energy }\end{array}$} \\
\hline & 60 & 90 & 98 \\
\hline exponential & 3 & 10 & 49 \\
\hline double exponential & al 3 & 6 & 13 \\
\hline normal & 2 & 3 & 4 \\
\hline log-normal & 3 & 4 & 6 \\
\hline rectangular & 2 & 2 & 12 \\
\hline
\end{tabular}

Table 3. Changes of the signal energy

In order to reflect obtained results in a real transmission conditions, two experimental channels in form of line pair connections on common printed circuit board (PCB) were tested by means of all defined pulse signals. First channel consists of parallel transmission lines with relatively large distance of $6 \mathrm{~mm}$, the second channel has similar structure but the distance between lines is only 0.5 $\mathrm{mm}$. Both channels are $30 \mathrm{~cm}$ long [14]. In the case of close lines, a significant crosstalk effect occurs, which affects real communication at high frequencies. The measured transfer functions meet low-pass filter functions with cutoff frequency of $1.2 \mathrm{GHz}$ (channel with crosstalk) or 1.8 $\mathrm{GHz}$ (channel without crosstalk). These cut-off frequencies in relation to the number of harmonics shown in Tabs. 1 to 3 give the possible highest fundamental frequency of periodic signals which repeat the defined pulses. In all periodic pulse signals, the duty cycle of $50 \%$ was assumed (first half of the period is a positive pulse and second half is zero signal). Table 4 shows the potential highest frequencies when keeping the features values at least 90\% of original values (ie acceptable distortion is $10 \%$ ). Table 4 represents the transmission channel without crosstalk. In case of crosstalk, all the values must be lowered by multiplication factor of 0.66 . 
Table 4. Highest fundamental frequency when feature values should be kept at least $90 \%$

\begin{tabular}{lccc}
\hline $\begin{array}{l}\text { Periodic } \\
\text { pulse signal }\end{array}$ & \multicolumn{3}{c}{$\begin{array}{l}\text { Highest fundamental } \\
\text { frequency in }\end{array}$} \\
\hline & height & shape & eneregy \\
\hline exponential & 90 & 45 & 200 \\
double exponential & 257 & 112 & 260 \\
normal & 600 & 450 & 900 \\
log-normal & 360 & 257 & 600 \\
rectangular & 1800 & 163 & 1800 \\
\hline
\end{tabular}

\section{CONCLUSION}

Considering all results given in Tables 1 to 4 , the best pulse waveform seems to be the normal pulse. For specific tasks, an optimal combination of pulse waveform and percentage threshold should be taken into account. In general, achieved results can help to better design electronic circuits working with pulse signals. Future work should focus on more variations of the used pulses and in addition new types of pulses should be analyzed so that more problems associated with pulses could be addressed. Furthermore, achieved results should be tested in practical applications.

\section{Acknowledgement}

Research described in this paper was financed by Czech Science Foundation (GACR) under grant 1518288S. For research, infrastructure of the SIX Center was used.

\section{REFERENCES}

[1] AdDison, P.-WALKER, J.-R. C. GUIDO, R. C.: Timefrequency analysis of biosignals, IEEE Engineering in Medicine and Biology Magazine 28 No. 5 (2009), 14-29.

[2] KetAtA, M.-LOUSSERT, A.-DHIEB, M.-LAhiAnI, M. GHARIANI, H.: The attenuation calculation of the energy signal of a Gaussian pulse propagating in the human body to detect the heart beat, International Review on Modelling and Simulations 6 No. 3 (2013), 937-945.

[3] AWAD, A. A.: The relationship between the photoplethysmographic waveform and systemic vascular resistance, Journal of clinical monitoring and computing 21 No. 6 (2007), 365-372.
[4] SEJDIC, E.-DJUROVIC, I.-JIANG, J. : Time-frequency feature representation using energy concentration: An overview of recent advances, Digital Signal Processing 19 No. 1 (2009), $154-188$.

[5] LI, J.-FAN, H.-XU, S.: Research on driving techniques of pulse signals for large capacity cascaded battery energy storage power conditioning system, High Voltage Engineering 41 No. 7 (2015), 2121-2126.

[6] VARAN, B.-YENER, A. : Incentivizing signal and energy cooperation in wireless networks, IEEE Journal on Selected Areas in Communications 33 No. 12 (2015), 2554-2566.

[7] PeVtsov, G.-YATsutsenko, A.-TROFimenko, Y.KARLOV, D.-BORTSOVA, M.: Theoretical basics of radar signals energy detection, Proc. IEEE International Conference on Mathematical Methods in Electromagnetic Theory (MMET) (2012), 324-327, Kharkiv, art. no. 6331269.

[8] FRAGKIADAKIS, A. et al: Signal processing techniques for energy efficiency, security, and reliability in the IoT domain, Modeling and Optimization in Science and Technology 8 (2016), 419-447.

[9] IFEACHOR, E. C.-JERVIS, B. W. : Digital Signal Processing, Englewood Cliffs: Prentice Hall, 2002.

10] SOLIMAN, S. S.-SRINATH, M. D. : Continuous and Discrete Signals and Systems, Englewood Cliffs: Prentice Hall, 1990.

[11] KRISHNAMOORTHY, K.: Handbook of Statistical Distributions with Applications. London: CRC Press (2016).

[12] GRCHENIG, K.: Foundations of Time-Frequency Analysis, New York: Springer, 2013.

[13] PTC Mathcad, Version 15 [Online]. Available: http://www.ptc. com/engineering-math-software/mathcad.

[14] SEVCIK, B.: Time-domain predistortion method based on raised cosine signaling in real transmission channels, Active and Passive Electronic Components, 2012, art. no. 596481.

Received 1 September 2016

Milan Sigmund received his master degree in 1984 in biomedical engineering and a doctoral degree in 1990 in speech signal processing, both from the Brno University of Technology. Currently, he is on the Faculty of Electrical Engineering and Communication at the same university. His research interests include signal processing with focus on speech signal analysis.

Lubomír Brančík received his master degree in 1985 in microelectronics and a doctoral degree in 1993 in measurements, both from the Brno University of Technology. Currently, he is on the Faculty of Electrical Engineering and Communication at the same university. His research interests include numerical techniques in electrical engineering, computer simulation, and signal integrity problems in electronic systems. 